\title{
Ultrasound-Assisted Removal Of Foreign Bodies In Oral And Maxillofacial Soft Tissues During Emergency Surgery: A Case Report
}

Dan Luo

Sichuan University West China College of Stomatology

Jinbo Zhou

Sichuan University West China College of Stomatology

Wei Tang

Sichuan University West China College of Stomatology

Wei Zeng ( 464751017@qq.com)

Sichuan University West China College of Stomatology https://orcid.org/0000-0002-4486-8798

\section{Research Article}

Keywords: foreign body, ultrasound technology, emergency surgery, case report

Posted Date: February 21st, 2022

DOI: https://doi.org/10.21203/rs.3.rs-970553/v1

License: @ (i) This work is licensed under a Creative Commons Attribution 4.0 International License.

Read Full License 


\section{Abstract}

Without accurate location and guidance, the removal of foreign bodies in the oral and maxillofacial tissues is difficult. Meanwhile, real-time assessment of whether all foreign bodies are removed during surgery is the key to a successful operation. Because of the long preparation time and the lack of realtime data, the present technologies such as navigation, guide template technology could not meet the requirements of emergency surgery and assess foreign body removal in real-time. In this report, a case of ultrasound technology assisting in the removal of foreign bodies in the oral and maxillofacial soft tissues during emergency surgery is presented. In conclusion, ultrasound technology can assist in the removal of foreign bodies in the oral and maxillofacial soft tissues, especially suitable for emergency surgery. It can realize accurate preoperative diagnosis and measurement, precise intraoperative localization, real-time guidance, measurement, and evaluation of foreign bodies.

\section{Introduction}

There are many types of foreign bodies in the oral and maxillofacial regions, which include shrapnel, broken dental needles, steel wire, glass, and other inorganic foreign bodies, as well as woodblocks, fish bones, gauzes, and seeds[1-3]. Foreign bodies in the oral and maxillofacial region could remain in tissues of different depths[4]. The traditional method mostly relies on the doctor's experience and the preoperative CT images[5], which often leads to failure to find the foreign body.

At present, the following techniques are commonly used to assist foreign body removal: guide template technology, surgical navigation, and augmented reality technology[6-8]. However, these techniques cannot obtain intraoperative data, resulting in real-time insufficiency and restricting their use in emergency surgery. Ultrasound technology is simple and can realize real-time intraoperative measurement and guidance. This report describes a case of using ultrasound technology to assist in the removal of non-metallic foreign bodies in soft tissues of the oral and maxillofacial region during emergency surgery.

\section{Case Presentation}

A 3-year-old boy had a wooden stick stabbing his left cheek and the presence of foreign bodies was confirmed by ultrasound examination in a local hospital. Then he was moved to the emergency department of West China Hospital of Stomatology. Physical examination revealed a sinus in the left cheek with surrounding skin redness and purulent secretions overflow. Preoperative multi-slice spiral computerized tomography (MSCT, $0.5 \mathrm{~mm}$ slice thickness) revealed an irregular high-density shadow on the outside of the left masseter muscle with an unclear boundary of the surrounding tissues( Fig. 1a).

As the child failed to cooperate, foreign bodies were removed under general anesthesia. At the beginning of the operation, the location, size, and quantity of the foreign bodies and their relationships with the surrounding blood vessels were first detected by the ultrasound(L14-5WU; Mindray Resona 5, Shenzhen, 
China) that can operate at 5 to $14 \mathrm{MHz}$. The real-time ultrasound showed an irregular hypoechoic area of about $2.51 \mathrm{~cm} \times 1.08 \mathrm{~cm}$ on the left cheek with a clear boundary, and four hyperechoic signals could be seen in the hypoechoic area, ranging in size from $0.12 \mathrm{~cm}$ to $1.44 \mathrm{~cm}$ (Fig. 1b-1d). Under the guidance of ultrasound, a total of 9 dark-brown wooden foreign bodies were removed during surgery(Fig. 1e). After clinical and ultrasound examinations confirmed repeatedly that the foreign bodies were removed completely, the wound was washed with a large amount of normal saline and then closed. In this case, 4 foreign bodies were detected before the operation, while 9 dark-brown wooden foreign bodies were removed. Among them, the smallest 4 pieces may be the debris separated from a larger wooden block, and the other piece with a size of $0.66 \mathrm{~cm}$ may be separated from the largest piece (Fig. 1f). The wound healed well without obvious redness and swelling when he was discharged from the hospital, and no obvious abnormality was found after 3 months.

\section{Conclusion}

Imaging techniques, such as CT, are currently applied in the diagnosis of foreign bodies in the oral and maxillofacial regions. In this report, the child was examined by ultrasound and MSCT before the operation. Ultrasound imaging revealed 4 foreign bodies, each with a clear boundary and a minimum size of $0.12 \mathrm{~cm}$ (Fig. 1d). Whereas MSCT could only show 2 foreign bodies, each with an unclear border, and the smallest foreign body was about $0.32 \mathrm{~cm}$ in size(Fig. 1a). The result proves that ultrasound could reveal foreign bodies more clearly, especially for those not developed on MSCT images, and smaller foreign bodies in soft tissues with their size and quantity accurately measured.

Ultrasound technology that can provide real-time data could realize real-time positioning and guidance during surgery and is easy to use. Some studies have reported the use of ultrasound technology to remove metal foreign bodies, such as steel wire and shrapnel, in the tongue and mouth floor area during surgery $[9,10]$. Metal foreign bodies are too hard to be broken by the external environment, while wood blocks and other organic foreign bodies are easily broken into small fragments.

Real-time assessment of whether all foreign bodies are removed during surgery is the key to the successful operation. Real-time measurement by ultrasound technology can help confirm whether wood blocks are broken. When the measured dimension is larger than the actual size, it will prove the remaining fragments. Meanwhile, ultrasound technology can provide real-time data during surgery to detect and locate the displaced or missing foreign bodies, determining whether all foreign bodies are removed. In this operation, we made full use of the advantages of ultrasound technology to successfully remove all the wooden foreign bodies on the patient's left cheek. In addition, the ultrasound instrument is relatively cheap, there is no radiation, the size is small, the environmental requirement for its installation is low, and this kind of portable equipment can be used in the operating room, which is especially suitable for emergency surgery.

In conclusion, ultrasound technology can assist in the removal of foreign bodies in the oral and maxillofacial soft tissues, especially suitable for emergency surgery. It can realize accurate preoperative 
diagnosis and measurement, precise intraoperative localization, real-time guidance, measurement, and evaluation of foreign bodies.

\section{Declarations}

\section{Ethical Approval and Informed Consent}

Consent for publication was obtained from the child's guardian. This study was approved by the ethics committee of West China Hospital of Stomatology.

\section{Conflicts of interest}

The authors declare that they have no conflict of interest.

\section{Funding}

Not applicable.

\section{Availability of Data and Material}

Not applicable.

\section{Code availability}

Not applicable.

\section{Authors' contributions}

Draft of the manuscript: Dan Luo, Wei Zeng, Wei Tang

Contribution and interpretation of radiological data: Jinbo Zhou

\section{Consent for publication}

Not applicable.

\section{Consent for participate}

Not applicable. 


\section{References}

1. Hirsch M, Droguett JP. A Plant Foreign Body of the Parotid Gland: The Seed That We Didn't SeeUltrasound Features and Review of the Literature. J Ultrasound Med. 2021 Oct;40(10):2255-2256.

2. Stein $\mathrm{K} \mathrm{M}$. Use of intraoperative navigation for minimally invasive retrieval of a broken dental needle[J]. J. Oral Maxillofac. Surg., 2015, 73(10): 1911-1916.

3. Kwak E J, Lee N J, Park W, et al. Foreign body removal assisted by an intraoral ultrasound probe[J]. Oral Radiol., 2019, 35(1): 73-76.

4. Ito R, Kubota K, Furudate $\mathrm{K}$, et al. Impalement of an unusual foreign body on the temporomandibular joint causing severe trismus[J]. Dent. Traumatol., 2016, 32(6): 514-516.

5. Lan L, He Y, An J, et al. Application of Computer-Aided Navigation Technology in the Extraction of Foreign Body From the Face[J]. J. Craniofac. Surg., 2020, 31(2): e166-e169.

6. Li P, Zhu H C, Huang D H. Detection of a metallic foreign body in the Wharton duct: A case report[J]. Medicine, 2018, 97(44).

7. Jain A, Gupta G, Grover M. Removal of an unusual neglected foreign body in infratemporal region using navigation[J]. J. Craniofac. Surg., 2017, 28(3): e219-e221.

8. Yao J, Zeng W, Zhou S, et al. Augmented reality technology could be an alternative method to treat craniomaxillofacial foreign bodies: a comparative study between augmented reality technology and navigation technology[J]. J. Oral Maxillofac. Surg., 2020, 78(4): 578-587.

9. Nimsky C, Ganslandt O, Cerny S, et al. Quantification of, visualization of, and compensation for brain shift using intraoperative magnetic resonance imaging[J]. Neurosurgery, 2000, 47(5): 1070-1080.

10. Kwak E J, Lee N J, Park W, et al. Foreign body removal assisted by an intraoral ultrasound probe[J]. Oral Radiol., 2019, 35(1): 73-76.

\section{Figures}

\section{Figure 1}

a Preoperative MSCT revealed two foreign bodies each with an unclear border and the smallest foreign body was about $0.32 \mathrm{~cm}$ in size. b An irregular hypoechoic area of about $2.51 \mathrm{~cm} \times 1.08 \mathrm{~cm}$. $\mathbf{c}$ Three hyperechoic signals could be seen and the sizes are $1.44 \mathrm{~cm}, 0.49 \mathrm{~cm}$, and $0.38 \mathrm{~cm}$, respectively. $\mathbf{d ~ A}$ hyperechoic signal with a size of $0.12 \mathrm{~cm}$. $\mathbf{e}$ The other piece of wood with a size of $0.66 \mathrm{~cm}$ was detected by ultrasound during the operation. $\mathrm{f} A$ total of 9 dark-brown wooden foreign bodies were removed

\section{Supplementary Files}

This is a list of supplementary files associated with this preprint. Click to download. 
- CAREchecklistEnglish2013.pdf 